\title{
Site of Fetal Membrane Rupture
}

National Cancer Institute

\section{Source}

National Cancer Institute. Site of Fetal Membrane Rupture. NCI Thesaurus. Code C117366.

The space that occupies the distance between the shortest free edge of the fetal membranes and the disc edge. 